\title{
ATUAÇÃO DO PSICOLÓGO NO HOSPITAL GERAL COM PACIENTES DE TENTATIVA DE SUICÍDIO: ESTUDO FENOMENOLÓGICO
}

\author{
Psychologists' work with suicide attempt patients in a general hospital: a \\ phenomenological study
}

\section{Actuación del psicólogo en el hospital general con pacientes que intentaron el suicidio: estudio fenomenológico}

\author{
Anna Karynne Melo \\ Universidade de Fortaleza - UNIFOR - Fortaleza (CE) - Brasil \\ Christina César Praça Brasil \\ Universidade de Fortaleza - UNIFOR - Fortaleza (CE) - Brasil \\ Ivina Alencar de Fiqueiredo \\ Universidade de Fortaleza - UNIFOR - Fortaleza (CE) - Brasil
}

Maisse Leoncio Catunda

Universidade de Fortaleza - UNIFOR - Fortaleza (CE) - Brasil

\section{Sarenne Pacheco Barbosa Carioca}

Universidade de Fortaleza - UNIFOR - Fortaleza (CE) - Brasil

\section{RESUMO}

Objetivo: Identificar a importância que os psicólogos atribuem à sua atuação em um hospital geral junto a pacientes com tentativas de suicídio. Métodos: Trata-se de uma pesquisa qualitativa de cunho fenomenológico, em que foram realizadas entrevistas a partir de uma pergunta disparadora, com o intuito de reconhecer como se dá a prática desse profissional com esse perfil de paciente. Resultados: Um dos dados obtidos reflete a importância da inserção do profissional de psicologia no hospital geral, pois ele é capaz de atuar em um meio multidisciplinar de modo a trazer reflexões para os outros profissionais e de promover uma escuta atenta, uma postura empática e compreensiva com um paciente que agiu contra a própria vida. Além disso, foi possibilitada reflexão acerca do papel do psicólogo no processo de humanização dentro do ambiente hospitalar. Conclusão: $\mathrm{A}$ atuação do psicólogo hospitalar com a tríade paciente - família - equipe é essencial no processo de humanização, sendo esse profissional quem vai intermediar essa relação.

Descritores: Suicídio; Atuação (Psicologia); Pesquisa Qualitativa.

\section{ABSTRACT}

Objective: To identify the importance assigned by psychologists to their work with suicide attempt patients in a general hospital. Methods: This is a qualitative phenomenological research that used interviews based on a trigger question used to assess the work of these professionals with such patients. Results: One of the data obtained demonstrated the importance of the inclusion of psychologists in a general hospital as they are capable of working in a multidisciplinary environment, sharing ideas with other professionals and promoting quality listening through an empathic behavior in order to understanding the patient who tried to take his/her own life. In addition, it was possible to ponder the role of the psychologist in the process of humanization within the hospital environment. Conclusion: The work of psychologists with the patient-family-team triad in the hospital is essential in the process of humanization, and they are the ones who will intermediate this relationship.

Descriptors: Suicide; Acting Out (Psychology); Qualitative Research. 


\section{RESUMEN}

Objetivo: Identificar la importancia atribuida por los psicólogos sobre su actuación en un hospital general con pacientes que intentaron el suicidio. Métodos: Se trata de una investigación cualitativa de abordaje fenomenológico en la cual se realizó entrevistas a partir de una pregunta guía para reconocer como se da la práctica de ese profesional con ese perfil de paciente. Resultados: Uno de los datos obtenidos refleja la importancia de la inserción del profesional de psicología en el hospital general pues lo mismo está capacitado para actuar en un medio multidisciplinario de modo a generar reflexiones para los demás profesionales y promover una escucha atenta, una postura empática y comprensiva con un paciente que fue en contra de su propia vida. Además de eso, se ha posibilitado una reflexión sobre el papel del psicólogo en el proceso de humanización dentro del ambiente hospitalario. Conclusión: La actuación del psicólogo hospitalario con la tríade paciente - familia - equipo es esencial para el proceso de humanización siendo ese profesional el que irá mediar esa relación.

Descriptores: Suicidio; Actuación (Psicología); Investigación Cualitativa.

\section{INTRODUÇÃO}

O suicídio constitui uma importante questão de saúde pública no mundo inteiro. Segundo a Organização Mundial da Saúde ${ }^{(1)}$, o suicídio é um fenômeno global, que chega a somar por ano cerca de 800.000 mortes em todo o mundo, dado que representa a realidade de uma morte por suicídio a cada 40 segundos.

O Brasil teve uma taxa nacional de suicídio de 5,01 por 100 mil habitantes, no ano de 2013, estando a cidade de Fortaleza, Ceará, na $5^{\mathrm{a}}$ posição entre as cidades brasileiras por número de suicídio, uma vez que foram registrados, em 2014, 154 óbitos ocasionados por automutilações ${ }^{(2,3)}$.

$\mathrm{O}$ ato suicida caracteriza-se pelo seu elevado grau de complexidade, diante da diversidade de motivações que levam um indivíduo a realizá-lo. Cada ser humano tem suas peculiaridades, mostrando que as vivências que podem fazer com que um indivíduo cometa um ato como esse se diferencia de pessoa a pessoa. É um momento de intenso sofrimento, diminuição do universo pessoal e sofrimentos causados, por exemplo, por uma crise de identidade, financeira, conjugal, no trabalho, na família. Dentre tantos motivos e causas, por mais que eles possam se repetir nos sujeitos, pode-se concluir que cada ser humano os vivencia de forma única e singular ${ }^{(4)}$.

Existem fatores de risco que atuam de forma acumulativa para o aumento de comportamentos suicidas nas pessoas, entre eles estão as dificuldades de acesso aos cuidados de saúde e à assistência necessária ${ }^{(1)}$. Dados da OMS ${ }^{(1)}$ apontam que apesar de o suicídio ser um ato individual resulta da associação de aspectos de natureza ambiental, social, fisiológica e genética. O Sistema de Informações sobre Mortalidade (SIM) mostram que há uma maior predisposição a suicídios em pessoas com mais idade, do sexo masculino, solteiras e com nível educacional mais baixo ${ }^{(5)}$. Diante de tantas variáveis, os transtornos mentais destacam-se como importantes fatores de risco para o suicídio(6), uma vez que estes vulnerabilizam os indivíduos e favorecem a concretização do ato.

Nesse contexto, os profissionais da saúde têm papel de destaque na identificação dos fatores de risco, na deteç̧ão de fatores preditivos ao ato suicida e no acolhimento de pessoas que apresentaram ideação ou tentativa suicida. Dentre estes profissionais, o psicólogo tem como função proporcionar um olhar diferenciado para o paciente; promover acolhimento baseado na aceitação incondicional, na empatia com o sujeito e com sua família; e possibilitar um clima favorável para a mudança na perspectiva do sujeito, da família e dos demais profissionais que atendem essa demanda(7).

Este estudo justifica-se pela necessidade de conhecer a realidade de atuação do psicólogo em hospitais que atendem pacientes que tentaram suicídio, uma vez que o conhecimento dessa realidade pode ampliar a inserção desses profissionais nos serviços de saúde, ampliando a qualidade da atenção aos pacientes e suas famílias.

O presente artigo visa identificar a importância que os psicólogos atribuem à sua atuação em um hospital geral junto a pacientes com tentativas de suicídio.

\section{MÉTODOS}

Realizou-se uma pesquisa qualitativa com base no método fenomenológico, que tem como artifício central, a redução fenomenológica. Por meio da redução fenomenológica há a possibilidade de se perceber e compreender o mundo, de enxergá-lo em sua realidade ${ }^{(8)}$. Com isso, é possível alcançar a realidade experiencial da inserção 
do psicólogo em um hospital geral que realiza atendimento a sujeitos com tentativa de suicídio, a realidade das necessidades dos pacientes e a realidade que compõe a rotina em um hospital geral.

A pesquisa foi realizada em maio de 2017, em um hospital geral e regional de Fortaleza, o qual é referência em procedimentos de alta complexidade, sendo o hospital privado com o maior número de leitos do Estado do Ceará.

A coleta de dados ocorreu por meio de entrevista não estruturada, que é quando o participante fala livremente sobre o tema ou questões apresentadas para aprofundamento ${ }^{(9)}$. Neste caso, a quantidade de participantes foi constituída pela equipe de psicologia do hospital, a qual é composta por quatro psicólogas.

Ressalta-se que as entrevistas foram realizadas individualmente por uma das pesquisadoras, a qual entrevistou cada uma das psicólogas no horário de trabalho, quando apresentavam disponibilidade de tempo. Nessa ocasião, após a explicação sobre a pesquisa e a assinatura do Termo de Consentimento e Livre Esclarecido (TCLE), apresentouse a seguinte questão disparadora: "De que modo se dá a sua atuação quando em contato com pacientes que cometeram tentativa de suicídio?”.

As entrevistas tiveram duração média de uma hora e foram registradas por meio de gravação em áudio. Além disso, a pesquisadora realizou observação participante e redigiu um diário de campo para facilitar a apreensão da realidade sob investigação. Em seguida, os dados obtidos por meio das gravações foram transcritos e alinhados aos registros escritos.

Destaca-se que a entrevista é uma estratégia muito pertinente para o alcance da compreensão do fenômeno, facilitando ao pesquisador a vivência do contexto investigado, uma vez que possibilita "explorar a experiência vivida e o sentido que o mundo vivido tem para o entrevistado ou entrevistados, e perceber como diferentes sujeitos experienciam certa condição comum a eles" (10, p. 265).

Nesse contexto, os entrevistados são denominados colaboradores ${ }^{(11)}$, uma vez que este autor defende que, na pesquisa fenomenológica, os sujeitos não são apenas fornecedores de dados, mas colaboram para que, em associação com o pesquisador, o assunto seja discutido. Acredita-se, nesse tipo de investigação, que é este colaborador quem melhor domina e conhece a sua experiência, cabendo ao pesquisador aprender com ele a respeito do assunto sobre o qual ele quer aprofundar seu saber ${ }^{(8)}$, havendo, assim, uma transformação mútua( ${ }^{(10)}$.

Para a análise e a interpretação dos dados, foi utilizado o método fenomenológico a partir da lente de MerleauPonty ${ }^{(8)}$, que traz a análise fenomenológica mundana como instrumento de pesquisa, por meio dos seguintes passos: divisão do texto nativo (transcrição literal da entrevista) em movimentos, segundo o tom da entrevista; análise descritiva do significado emergente do movimento e o sair dos parênteses.

No método fenomenológico a redução é um movimento que não finaliza e que, nesse contexto, o investigador não consegue abandonar totalmente os seus pressupostos e idealizações a respeito do objeto de pesquisa ${ }^{(8)}$. Assim, nos resultados, é comum que o "pesquisador saia do parêntese, trazendo a lupa para a sua hipótese, assumindo-se integralmente como pesquisador mundano, dialogando com os resultados da pesquisa e, sobretudo, posicionandose diante dos resultados" (10, p. 265).

A partir desse procedimento, neste estudo, identificaram-se três temáticas de análise, as quais são apresentadas e discutidas nos resultados, a partir de uma abordagem que possibilita esclarecer sobre a problemática em questão.

Ressalta-se que, para a preservação das identidades das participantes, as falas que ilustram os resultados foram apresentadas com nomes fictícios.

Esta pesquisa foi desenvolvida em concordância com os padrões éticos, respeitando a Resolução 466/12 do Conselho Nacional de Saúde, cujo projeto foi aprovado pelo Comitê de Ética da Universidade de Fortaleza sob o Parecer $\mathrm{n}^{\circ}$ 2.033.030.

\section{RESULTADOS E DISCUSSÃO}

Com as entrevistas realizadas, foram evidenciadas três temáticas que abordam aspectos relevantes sobre a inserção do psicólogo em um hospital geral e o desenvolvimento de uma prática de qualidade junto a pacientes que tentaram suicídio, quais sejam: atendimento ao paciente e à família; papel de mediação de conflitos internos e externos; e trabalho de preparação da equipe multidisciplinar.

A partir da conceituação do que é suicídio e da agressão aplicada a si mesmo com o intuito de autodestruição, algumas situações vivenciadas por indivíduos em geral, mas principalmente por aqueles que demonstram certos aspectos e sintomas que levam a crer na possibilidade de tendências suicidas, podem, muitas vezes, não ser consideradas por um viés suicida. Entretanto, quando há uma ampliação do olhar, pode-se entender que essas 
situações apontam para um modo de ser perpassado pelo desinteresse, pela falta de cuidado consigo mesmo. Ou seja, não apenas atos extremados devem ser postos em foco, mas toda uma gama de gestos que evidenciam o descompromisso da pessoa com ela própria pode alarmar para tentativas de suicídio(12,13).

Sobre o atendimento ao paciente e à família, as psicólogas relataram o modo como o atendimento é realizado. Para elas, o psicólogo hospitalar tem que disponibilizar atendimento diante desse sujeito que está precisando de ajuda, tendo como base a utilização de uma atitude empática, como forma de tentar entender as motivações que levaram o sujeito ao ato. Além disso, é fundamental proporcionar reflexões ao paciente para que ele pense sobre o seu ato:

"Realmente tem que disponibilizar atendimento psicológico. Se chegam ao ponto desse sofrimento, dessa angustia ser tão grande, tão grave ao ponto de acabar com a própria vida, então é porque ele está precisando de ajuda." (Lia)

"No primeiro momento é o sentimento de empatia... É primeiro tentar entender o que levou aquela pessoa a essa tentativa." (Alana)

“... a família para a gente é muito importante. Para mim, essa escuta da família é muito necessária." (Ana)

O cuidado psicológico é essencial quando se trata de suicídio. As tentativas de suicídio não devem ser supervalorizadas nem desvalorizadas, sendo necessário entendê-las e acolhê-las verdadeiramente ${ }^{(14)}$. Nesse cenário, o psicólogo precisa estar aberto e preparado para receber tanto os pacientes e seus familiares, sem pré-julgamentos ou juízos de valor. Esse profissional torna-se essencial no momento de acolhimento ao ambiente hospitalar, pois o vínculo estabelecido nessa ocasião será determinante para o êxito do atendimento de toda a equipe de saúde.

O manejo dos pacientes que passaram por tentativas de suicídio requer respeito, disponibilidade, trabalho contínuo que abranja as dores, tolerância às frustações e à falta de sentido que o outro atribui à sua vida, e trabalho interdisciplinar com a família e outros profissionais. E, o trabalho do psicólogo com o tema suicídio envolve questões existenciais, visto que se tem como queixas, muitas vezes, sentimentos de solidão, tédio, medo, incertezas e falta de prazer na vida ${ }^{(15,16)}$. Quanto ao papel de mediação de conflitos internos e externos do psicólogo, este deve realizar uma escuta, orientando, desmitificando e propondo reflexões ${ }^{(17)}$ :

“... o foco desse atendimento é no sentido de o que ele vê nessa vida, o que faz sentido para ele estar vivo. Trabalhar essas coisas, pessoas ou situações que prendam ele a essa vida. O que faz sentido para ele nessa vida e também o outro olhar dessa vida, porque, muitas vezes, o que a gente percebe no paciente suicida é que ele tem uma visão muito para o lado negativo..." (Alana)

Considerando outras características da psicologia hospitalar citadas pelas psicólogas, é válido ressaltar a relevância da teoria de crise como um olhar utilizado nas intervenções com os pacientes hospitalizados. A teoria da crise pode ser compreendida "como um estado psicológico transitório, em que o fator desencadeante é o desequilíbrio entre a percepção da dificuldade, a importância do problema e os recursos disponíveis para solução"(12, p.100). Nesse sentido, as profissionais entrevistadas apontam para técnicas interventivas focadas para a prevenção de novos episódios suicidas, a partir da tentativa de se desenvolver um estado emocional mais tolerável à realidade do paciente.

Uma das psicólogas (Alana) reflete sobre o que de imediato deve ser compreendido quando se tem contato com um paciente que passou por uma tentativa de suicídio. A atuação do psicólogo vai caminhar justamente na tentativa de promover um equilíbrio na percepção entre as dificuldades existentes. Afinal, a vida não é só feita de problemas. Por isso, perguntar sobre o que faz sentido na vida é produzir uma reflexão a respeito do que se tem de bom; a importância dos problemas e os recursos disponíveis para solução.

Uma das psicólogas acredita que existem questionamentos fundamentais quando se atende pacientes que cometeram tentativa de suicídio para que se possa conhecer ou, pelo menos inferir as motivações para o ato, tais como:

“... as perguntas chaves são: ver o histórico do paciente, se tinha histórico psiquiátrico e já tratava ou não.... Como é essa relação familiar? O que realmente pode ter motivado esse ato? [...] Saber se há grande possibilidade de ele fazer de novo ou não..." (Lia)

Para intervenções nesses casos também é de fundamental valor que sejam consideradas situações como: a urgência do atendimento, a estrutura de personalidade do paciente, o histórico e o diagnóstico atual, as relações interpessoais, as perdas, as doenças físicas, entre outros aspectos ${ }^{(18)}$.

Por vezes, o psicólogo deve intermediar a internação do paciente em instituições especializadas, quando necessário. Outrossim, este profissional deve trabalhar para a ampliação do sistema de apoio, procurando ajudar a 
família na compreensão de que a pessoa que tenta ou comete suicídio pode não desejar a morte, e sim viver de outra maneira ${ }^{(19)}$. Nesse sentido, é fundamental trabalhar em conjunto com psiquiatras, numa abordagem multidisciplinar, uma vez que, a partir dos discursos das psicólogas, existe uma parceria de grande valia delas com o psiquiatra no atendimento aos pacientes suicidas no hospital geral( ${ }^{(20)}$ :

"O psiquiatra ajuda a gente nesse sentido, de propriamente diagnosticar ou ter pelo menos uma suposição, uma hipótese diagnóstica para nortear também a nossa atuação." (Tábata)

"Todos esses pacientes que entram por tentativa [de suicídio] a gente faz avaliação deles. Então a gente avalia, vê o estado de humor, questão de possibilidades claras de novas tentativas, avalia ideação, tudo isso. [...] É perguntado para família se ele já tem acompanhamento psiquiátrico, se sim a gente pergunta se esse psiquiatra pode vir, se não a gente aciona o psiquiatra da casa. Então esse é mais ou menos o fluxo." (Lia)

É fundamental o psicólogo trabalhar em conjunto com psiquiatras, numa abordagem multidisciplinar, no atendimento a pacientes que tentaram cometer suicídio, uma vez que abordagens terapêuticas e medicamentosas, muitas vezes, precisam estar associadas para possibilitar a obtenção de melhores resultados ${ }^{(21)}$.

Diante da complexidade dos casos, o atendimento do psicólogo no hospital deve levar em consideração a tríade paciente - família - profissionais. Isto reflete a importância que tem esse profissional também no atendimento e no suporte à equipe de saúde. $O$ trabalho do psicólogo junto à equipe de saúde no contexto dos pacientes suicidas contempla todos os profissionais envolvidos e ajuda no enfrentamento do contexto conturbado e urgente em que eles trabalham.

A partir do entendimento do que envolve um atendimento de urgência, das vivências dos profissionais, pacientes e familiares, a psicologia hospitalar vai atuando para a promoção de saúde do paciente e a prevenção de agravos, voltando-se para a atuação de excelência e saudável dos profissionais e para a aceitação e a compreensão da família $^{(22)}$. A rede social de apoio, como a família, amigos e pessoas próximas, é também relevante, pois é por meio dela que os indivíduos passam a acreditar que recebem cuidados, que são amados, queridos e estimados, favorecendo a autoestima de cada um e, por consequência, transformando esses atos em situações e sentimentos mútuos ${ }^{(23)}$.

As famílias e a equipe podem contribuir de modo positivo com o paciente, a partir de uma escuta facilitadora, da compreensão da angústia do indivíduo e da possível falta de vontade de viver. É válido destacar, que há pequenos gestos que passam despercebidos e servem de alerta para uma possível tentativa de suicídio, o que pode ser mostrado pelo psicólogo hospitalar aos familiares desse paciente ${ }^{(24)}$.

A falta de sentido da vida pode ser um significativo componente na tomada de uma decisão violenta de se matar. É preciso não deixar o paciente sozinho quando há o potencial de suicídio, devendo este ser acompanhado no dia a dia ${ }^{(21)}$.

Essa realidade aponta para a necessidade do profissional de psicologia dentro de uma emergência hospitalar. O trabalho realizado pelo psicólogo com toda a equipe multiprofissional passa a ter como objetivo, também, a promoção de uma mudança no olhar direcionado ao paciente suicida, evidenciando para o fato de que o atendimento realizado a esse paciente hoje influencia, de modo considerável, na reprodução de outro ato ${ }^{(22)}$ :

"... é importante ressaltar que no hospital esse cuidado deve ser também com a equipe. Essa equipe que cuida, que está ali para cuidar desse paciente, mas um profissional que, muitas vezes, não teve esse preparo." (Alana)

Para que ocorra um atendimento psicológico promotor de crescimento do paciente, é necessário que o psicólogo se disponibilize e, que entenda que o seu local de atendimento é qualquer lugar que o paciente esteja e lhe demande. Entende-se que no passado e no futuro não existe ação, que a experiência se dá no presente, ou seja, no aqui e agora que é dito pelo paciente. Para o paciente, o agora é permeado pelas lembranças ou por possibilidade, e o psicólogo que atua nessa situação de crise deve supor que no contexto de tentativa de suicídio se configura o passado repleto dos porquês desse ato e com um futuro incerto ${ }^{(26)}$.

Portanto, o atendimento deve se encaminhar no aqui e agora, agindo no tempo presente e no que pode ser feito para o paciente naquele momento, mesmo que seja um atendimento único.

\section{CONSIDERAÇÕES FINAIS}

Visto que a hospitalização traz para o paciente interno uma variedade de obrigações médicas, limitações das vontades e dos papeis do ser, perdas de autonomia e limitações sociais e físicas, questiona-se sobre a qualidade 
de vida desse paciente, sobre a sua condição humana nesse ambiente que nasceu para cuidar, mas que muitas vezes não se propõe a contemplar as singularidades daqueles que devem ser cuidados.

$\mathrm{Na}$ instituição em questão, demasiada são as ações desenvolvidas pelas profissionais da Psicologia que visam uma real humanização no processo de internação e, além disso, que elas conseguem atingir de fato os outros profissionais para a importância de se considerar e de se ter uma atuação não mecanizada para com o outro.

É válido entender as práticas psicológicas que se inserem no hospital e compreender que elas vêm propor uma visão oposta à que antes era pensada sobre o processo saúde-doença. Anteriormente os Médicos e Enfermeiros se utilizavam do modelo biomédico em que se tinha a compreensão de que a saúde é a ausência de doença, ou seja, esse novo profissional da Psicologia passa a trazer um olhar diferenciado, em que a doença passa a fazer parte de um homem que está contextualizado em uma realidade biopsicossocial. Realidade essa que passa a considerar questões sociais e emocionais no processo de adoecimento biológico.

A atuação do Psicólogo hospitalar aqui, portanto, torna-se essencial no processo de humanização, sendo ele o componente que ouve o grito de dor do paciente de modo que este seja não somente escutado, mas, sobretudo, compreendido em toda a sua dimensão humana. É preciso que o seu desejo de morrer não seja julgado e sim compreendido dentro da sua existência humana.

Entender a relevância do Psicólogo hospitalar no hospital geral para atendimento de pacientes com tentativa de suicídio é complexa. Nas entrevistas realizadas foi discutido e descrito sobre a prática do Psicólogo hospitalar que atua em um hospital geral, tendo como foco o suicídio, pois há aumento de número de casos de tentativa de suicídio.

Destaca-se que nesse momento de grande aumento dos índices de tentativa de suicídio e de suicídio, esta pesquisa traz a contribuição e um ineditismo na seara das problematizações no campo da saúde e na possibilidade da promoção nos cuidados com pessoas nessas situações.

\section{AGRADECIMENTOS E CONFLITOS DE INTERESSE}

Autores agradecem à Universidade de Fortaleza e ao Hospital da pesquisa pela colaboração na realização da mesma.

O artigo não apresenta conflito de interesses.

\section{REFERENNCIAS}

1. Organização Mundial da Saúde. Departamento de saúde mental e abuso de substâncias. Prevenção de suicídio: um recurso para conselheiros [Internet]. Genebra: OMS; 2006. [acesso em 2018 Ago 05]. Disponível em: http:// www.who.int/mental_health/media/counsellors_portuguese.pdf

2. Departamento de Informática do SUS - Datasus. Óbitos por causas externas - Brasil [Internet]. Brasília: Ministério da Saúde; 2016 [acesso em 2018 Ago 05]. Disponível em: http://tabnet.datasus.gov.br/cgi/deftohtm.exe?sim/ cnv/ext10br.def

3. Moreira RMM, Félix TA, Flôr SMC, Oliveira EN, Albuquerque JHM. Análise epidemiológica dos óbitos por suicídio. Sanare (Sobral). 2017;16(Supl 1):29-34.

4. Botega NJ. Crise suicida: avaliação e manejo. Porto Alegre: Artmed; 2015.

5. Ribeiro JF, Mascarenhas TB, Araújo ACBS, Coelho DMM, Pedra Branca SB, Coelho DMM. Perfil sociodemográfico da mortalidade por suicídio. Rev Enferm UFPE. 2018;12(1):44-50.

6. Botega NJ. Comportamento suicida: epidemiologia. Psicol USP. 2014;25(3):231-6.

7. Gondim DSM. A intervenção da psicologia: tentativas de suicídio e urgência hospitalar. Rev Científica FMC. 2015;10(2):12-6.

8. Moreira V. O método fenomenológico de Merleau-Ponty como ferramenta crítica em psicopatologia. Psicol Reflex Crít. 2004;17(3):447-56.

9. Minayo MCS. O desafio do conhecimento. São Paulo: Hucitec; 2014.

10. Siani SR, Correa DA, Las Casas AL. Fenomenologia, método fenomenológico e pesquisa empírica: o instigante universo da construção de conhecimento esquadrinhada na experiência de vida. Rev Adm UNIMEP. 2016;14(1):193219. 
11. Amatuzzi MM. Pesquisa fenomenológica em Psicologia. In: Holanda AF, Bruns MAT, organizadores. Psicologia e fenomenologia: reflexões e perspectivas. Campinas: Alínea; 2000. p. 16-7.

12. Rocha MAS, Boris GDJB, Moreira V. A experiência suicida numa perspectiva humanista-fenomenológica. Rev Abordagem Gestál. 2012;18(1):69-78.

13. Bahia CA, Avanci JQ, Pinto LW, Minayo MCS. Lesão autoprovocada em todos os ciclos da vida: perfil das vítimas em serviços de urgência e emergência de capitais do Brasil. Ciênc Saúde Colet. 2017;22(9):2841-50.

14. Bastos RL. Suicídios, psicologia e vínculos: uma leitura psicossocial. Psicol USP. 2009;20(1):67-92.

15. Moreira DL, Martins MC, Gubert FA, Sousa FSP. Perfil de pacientes atendidos por tentativa de suicídio em um centro de assistência toxicológica. Ciênc Enferm. 2015;21(2):63-75.

16. Vieira LP, Santana VTP, Suchara EA. Caracterização de tentativas de suicídios por substâncias exógenas. Cad Saúde Colet (Rio de J). 2015;23(2):118-23.

17. Fukumitsu KO. O psicoterapeuta diante do comportamento suicida. Psicol USP. 2014;25(3):270-5.

18. Andreoli PBA, Caiuby AVS, Lacerda SS. Psicologia hospitalar. Barueri: Manole; 2013. (Manuais de especialização Einstein).

19. Jaramillo-Moreno RA. Resiliencia familiar, comprensión, campos de aplicación, aportes y desafios. Diversitas Perspectiv Psicol. 2017;13(2):255-66.

20. Silva MVS, Miranda GBN, Andrade MA. Sentidos atribuídos à integralidade: entre o que é preconizado e vivido na equipe multidisciplinar. Interface Comun Saúde Educ. 2017;21(62):589-99.

21. Fukumitsu KO, Kovács MJ. Especificidades sobre processo de luto frente ao suicídio. Psico (Porto Alegre). 2016;47(1):3-12.

22. Aguiar MSV, Cavalcante LA, Ramos IWS, Holanda TCM. A atuação do psicólogo hospitalar no atendimento a pacientes com doenças cardiovasculares: revisão de literatura. Div Práticas Psicologia. 2018;1(1):65-70.

23. Cobb S. Social Support as a moderator of life stress. Psychosom Med. 1976;38(5):300-314.

24. Cassorla RMS. O impacto dos atos suicidas no médico e na equipe de saúde. In: Cassorla RMS, organizador. Do suicídio: estudos brasileiros. Campinas: Papirus; 1991. p. 149-65.

25. Vidal CEL, Gontijo ED. Tentativas de suicídio e o acolhimento nos serviços de urgência: a percepção de quem tenta. Cad Saúde Colet (Rio de J). 2013;21(2):108-14.

26. Azevêdo AVS, Crepaldi MA. A psicologia no hospital geral: aspectos históricos, conceituais e práticos. Estud Psicol (Campinas). 2016;33(4):573-85.

\section{Endereço para correspondência:}

Anna Karynne Melo

Universidade de Fortaleza - UNIFOR

Programa de Pós-Graduação em Saúde Coletiva

Av. Washington Soares, 1321, Bloco S, Sala 1

Bairro: Edson Queiroz

CEP: 60.811-905 - Fortaleza - CE - Brasil

E-mail: karynnemelo@unifor.br 